\title{
The contribution of chiral three-body forces to the monopole component of the effective shell-model Hamiltonian
}

\author{
Y. Z. Ma, ${ }^{1}$ L. Coraggio, ${ }^{2}$ L. De Angelis, ${ }^{2}$ T. Fukui, ${ }^{2}$ A. Gargano, ${ }^{2}$ N. Itaco, ${ }^{2,3}$ and F. R. Xu ${ }^{1}$ \\ ${ }^{1}$ School of Physics, and State Key Laboratory of Nuclear Physics and Technology, \\ Peking University, Beijing 100871, China \\ ${ }^{2}$ Istituto Nazionale di Fisica Nucleare, \\ Complesso Universitario di Monte S. Angelo, Via Cintia, I-80126 Napoli, Italy \\ ${ }^{3}$ Dipartimento di Matematica e Fisica, Università degli Studi della Campania "Luigi Vanvitelli", \\ viale Abramo Lincoln 5 - I-81100 Caserta, Italy
}

\begin{abstract}
We present a study of the role played by realistic three-body forces in providing a reliable monopole component of the effective shell-model Hamiltonian. To this end, starting from a nuclear potential built up within the chiral perturbation theory, we derive effective shell-model Hamiltonians with and without the contribution of the three-body potential and compare the results of shell-model calculations with a set of observables that evidence shell-evolution properties. The testing ground of our investigation are nuclei belonging to $f p$ shell, since the shell-evolution towards shell closures in ${ }^{48} \mathrm{Ca}$ and ${ }^{56} \mathrm{Ni}$ provides a paradigm for shell-model Hamiltonians. Our analysis shows that only by including contributions of the three-body force the monopole component of the effective shell-model Hamiltonian is then able to reproduce the experimental shell evolution towards and beyond the closure at $N=28$.
\end{abstract}

PACS numbers: 21.60.Cs, 21.30.Fe, 21.45.Ff, 27.40.+z

\section{INTRODUCTION}

The evolution of the nuclear spectroscopic properties along isotopic and isotonic chains, towards the formation of magic numbers, is the feature that reveals the central role of the nuclear shell model (SM) and its success during the past 70 years $[1]$. Consequently, it is very desirable that effective Hamiltonians, which are employed to study the nuclear structure in the framework of the shell model, should be able to reproduce the observed shell evolution and closures.

Zuker and coworkers have extensively investigated the properties of the two-body matrix elements (TBMEs) of the residual interaction derived from realistic potentials by way of many-body perturbation theory [4, and, having performed a multipole decomposition of realistic SM Hamiltonians, have shown that their monopole component needs to be modified in order to reproduce the evolution of shell closures as a function of the number of valence nucleons [5 7]. They have inferred that this should trace back to the lack of a three-nucleon force $(3 \mathrm{NF})$ in the nuclear realistic potentials employed to derive the effective SM Hamiltonian $H_{\text {eff }}$, affecting its monopole component that, consequently, has to be corrected [8].

Extensive direct investigations about the role of $3 \mathrm{NFs}$ in realistic $H_{\text {eff }} \mathrm{s}$ have been carried out by Schwenk and coworkers, who have performed studies of oxygen 9.13 and calcium [11, 13,15] isotopic chains. In the aforementioned works, the $H_{\text {eff }}$ blue have been derived starting from nuclear potentials built up within the chiral perturbative expansion and softened by way of the $V_{\mathrm{low}-\mathrm{k}}$ technique [16, 17] or the similarity renormalization-group (SRG) approach [18, 19], and the results have supported the need of introducing three-body forces to reproduce the experimental behavior of the ground-state and yrast excitation energies as a function of the valence-nucleon number.

In order to investigate the role played by three-body forces in driving the shell evolution, we have found inspiration from the calculation of the effective single-particle energies (ESPEs) for $p$-shell nuclei, whose results we have presented in Ref. 20. More precisely, we have found that the ESPEs calculated from the $H_{\text {eff }}$ that includes contributions from both two- and three-body chiral potentials, provide a constant energy-splitting of the spin-orbit partners $0 p_{3 / 2}, 0 p_{1 / 2}$ as a function of the mass number $A$. This splitting characterizes the correct reproduction of the subshell closure at $Z, N=6$ observed in ${ }^{12} \mathrm{C}$, at variance with the result we have obtained omitting the contribution of the $3 \mathrm{NF}$. As a matter of fact, the relative ESPE rapidly drops down if only the two-nucleon force (2NF) is included, and becomes even negative around $A=8$. Then, the reproduction of the shell closure deteriorates, namely the observed energy of the ${ }^{12} \mathrm{C}$ yrast $J^{\pi}=2^{+}$state is underestimated by $\sim 1 \mathrm{MeV}$.

Since the ESPE of a level is calculated in terms of the bare single-particle (SP) energy and the monopole part of the TBMEs [21, it is clear that the above mentioned results point to an intimate relationship between $3 \mathrm{NF}$ and the monopole component of $H_{\text {eff }}$.

On the above grounds, we devote the present paper to studying this connection choosing, as a testing ground, the nuclei belonging to the $f p$ shell, namely those that can be described in terms of the degrees of freedom of valence nucleons outside doubly-closed ${ }^{40} \mathrm{Ca}$, interacting in the model space composed by $0 f 1 p$ orbitals. This region represents a paradigm to investigate the shell evolution within the shell model, since, as is well-known, the spinorbit component of the SM mean field separates the $0 f_{7 / 2}$ orbital from the others leading to the appearance of the 
magic number $Z, N=28$ and, consequently, of the two doubly-magic nuclei ${ }^{48} \mathrm{Ca}$ and ${ }^{56} \mathrm{Ni}$.

The starting point of our calculation is a nuclear potential based on chiral perturbation theory (ChPT) 22, 23], a choice that is motivated by two main considerations.

a) First, within this class of potentials long-range forces are ruled by the symmetries of low-energy quantum chromodynamics (QCD) - in particular the spontaneously broken chiral symmetry - and the short-range dynamics is absorbed into a complete basis of contact terms that are proportional to low-energy constants (LECs) fitted to two-nucleon data.

b) The second major characteristic of ChPT is that nuclear $2 \mathrm{NF}$ and many-body forces are generated on an equal footing [23 25], since most interaction vertices that appear in the $3 \mathrm{NF}$ and in the fournucleon force (4NF) also occur in the $2 \mathrm{NF}$.

For the sake of completeness, we point out that, as in Ref. 20, a high-precision $2 \mathrm{NF}$ potential derived within the ChPT at next-to-next-to-next-to-leading order $\left(\mathrm{N}^{3} \mathrm{LO}\right)$ [23. 26] is considered in our calculation, without any renormalization of its high-momentum components, juxtaposed with a $\mathrm{N}^{2} \mathrm{LO} 3 \mathrm{NF}$ potential, since this manybody contribution appears from this order on. Nowadays, these potentials are widely employed in nuclear theory aiming to link the fundamental theory of strong interactions, the QCD, to nuclear many-body phenomena.

Then, the $H_{\text {eff }}$ for systems with one- and two-valence nucleons outside the ${ }^{40} \mathrm{Ca}$ core are derived by way of the energy-independent linked-diagram perturbation theory 27, where $2 \mathrm{NF}$-vertices diagrams are included up to third order and contributions of $3 \mathrm{NF}$ up to first order in the perturbative expansion.

For those nuclei with a number of valence nucleons larger than 2 - we will report calculations for $Z=20$, $22,24,26$, and 28 up to $N=40$ - the effect of manybody correlations is taken into account by including the contributions of three-body diagrams calculated at second order in perturbation theory [28]. These correlations arise from the interaction via the two-body force of the valence nucleons with excitations outside the model space [29]. Since our SM code cannot manage three-body Hamiltonians, we have derived a density-dependent twobody contribution at one-loop order from the three-body correlation diagrams, summing over the partially-filled model-space orbitals.

A description of the perturbative approach to the derivation of our effective SM Hamiltonian is reported in Section II, where the perturbative properties are also discussed in some detail. In Section III we introduce first the results of the calculation of the ESPEs, in order to analyze the properties of the monopole component of the effective Hamiltonians, obtained with and without the contribution from a chiral 3NF. Then, we compare the results of the full diagonalization of these $H_{\text {eff }} \mathrm{s}$ with observables that are sensitive to the shell evolution of $f p$ isotopic chains. We focus on the evolution of collectivity in $N=28$ isotones too, that is a key point to evaluate the balance between the monopole and quadrupole components of the effective SM Hamiltonian. Finally, in Section IV we draw the conclusions of our study and the outlook of our future work.

\section{OUTLINE OF CALCULATIONS}

As mentioned in the Introduction, we choose, as $2 \mathrm{NF}$, the chiral $\mathrm{N}^{3} \mathrm{LO}$ potential derived by Entem and Machleidt in Ref. [26], and as $3 \mathrm{NF}$ a chiral $\mathrm{N}^{2} \mathrm{LO}$ potential, which shares the regulator function of a nonlocal form and some of the LECs with the 2NF. It is worth pointing out that the $\mathrm{N}^{2} \mathrm{LO} 3 \mathrm{NF}$ is composed of three components, namely the two-pion $(2 \pi)$ exchange term $V_{3 N F}^{(2 \pi)}$, the one-pion $(1 \pi)$ exchange plus contact term $V_{3 N F}^{(1 \pi)}$, and the contact term $V_{3 N F}^{(\mathrm{ct})}$.

For the sake of consistency, the $c_{1}, c_{3}$, and $c_{4}$ LECs appearing in $V_{3 \mathrm{NF}}^{(2 \pi)}$, are the same as those in the $\mathrm{N}^{3} \mathrm{LO}$ $2 \mathrm{NF}$, their values being determined by the renormalization procedure that fits the nucleon-nucleon $(N N)$ data 23 .

Moreover, the $3 \mathrm{NF} 1 \pi$-exchange and contact terms are characterized by two extra LECs (known as $c_{D}$ and $c_{E}$, respectively), which cannot be constrained by two-body observables, but need to be determined by reproducing observables in systems with mass $A>2$.

We adopt the same $c_{D}, c_{E}$ values employed in Ref. [20], namely $c_{D}=-1$ and $c_{E}=-0.34$, that have been determined by way of no-core shell model (NCSM) calculations [30, 31. More precisely, in Ref. 30] it has been identified a set of observables in light $p$-shell nuclei that are strongly sensitive to the $c_{D}$ value in order to fix it, then $c_{E}$ has been constrained to reproduce the binding energies of the $A=3$ system.

Details about the calculation of our $3 \mathrm{NF}$ matrix elements in the harmonic-oscillator (HO) basis are reported in Appendix of Ref. [20]. Note that the Coulomb potential is explicitly taken into account in our calculations.

In the same paper, a comprehensive description of the derivation of our effective SM Hamiltonians for oneand two-valence nucleon systems, starting from $2 \mathrm{NF}$ and $3 \mathrm{NF}$, can also be found, while in the following we present only a short summary.

As mentioned before, our $H_{\text {eff }}$ is derived in the model space spanned by the four $0 f 1 p$ proton and neutron orbitals outside doubly-closed ${ }^{40} \mathrm{Ca}$.

To this end, an auxiliary one-body potential $U$ is introduced in order to break up the Hamiltonian $H$ for a system of $A$ nucleons as the sum of a one-body term $H_{0}$, which describes the independent motion of the nucleons, 
and a residual interaction $H_{1}$ :

$$
\begin{aligned}
H= & \sum_{i=1}^{A} \frac{p_{i}^{2}}{2 m}+\sum_{i<j=1}^{A} V_{i j}^{2 \mathrm{NF}}+\sum_{i<j<k=1}^{A} V_{i j k}^{3 \mathrm{NF}}= \\
= & T+V^{2 \mathrm{NF}}+V^{3 \mathrm{NF}}=(T+U)+ \\
& +\left(V^{2 \mathrm{NF}}-U\right)+V^{3 \mathrm{NF}}=H_{0}+H_{1}^{2 \mathrm{NF}}+H_{1}^{3 \mathrm{NF}}
\end{aligned}
$$

In our calculation we use the HO potential, $U=\frac{1}{2} m \omega^{2} r^{2}$, with an oscillator parameter $\hbar \omega=11 \mathrm{MeV}$, according to the expression [32] $\hbar \omega=45 A^{-1 / 3}-25 A^{-2 / 3}$ for $A=40$.

Once $H_{0}$ has been introduced, the reduced model space is defined in terms of a finite subset of $H_{0}$ 's eigenvectors. The diagonalization of the many-body Hamiltonian in Eq. (1) within the infinite Hilbert space, that it is obviously unfeasible, is then reduced to the solution of an eigenvalue problem for an effective Hamiltonian $H_{\text {eff }}$ in a finite space.

Our approach to the derivation of $H_{\text {eff }}$ is the timedependent perturbation theory [27, 33, 34]. Namely, $H_{\text {eff }}$ is expressed through the Kuo-Lee-Ratcliff (KLR) foldeddiagram expansion in terms of the vertex function $\hat{Q}$-box, which is composed of irreducible valence-linked diagrams [35, 36]. We include in the $\hat{Q}$-box one- and two-body Goldstone diagrams through third order in $H_{1}^{2 \mathrm{NF}}$ and up to first order in $H_{1}^{3 \mathrm{NF}}$.

In Fig. 1 we report the contribution at first order in perturbation theory to the single-particle component of the $\hat{Q}$-box of a three-body potential, whose explicit expression is:

$$
\begin{aligned}
& \left\langle j_{a}\left|1 b_{3 N}\right| j_{a}\right\rangle= \\
& \sum_{\substack{h_{1}, h_{2} \\
J_{12} J}} \frac{\hat{J}^{2}}{2 \hat{j}_{a}^{2}}\left\langle\left[\left(j_{h_{1}} j_{h_{2}}\right)_{J_{12}}, j_{a}\right]_{J}\left|V_{3 N}\right|\left[\left(j_{h_{1}} j_{h_{2}}\right)_{J_{12}}, j_{a}\right]_{J}\right\rangle .
\end{aligned}
$$

The expression of the first-order two-body diagram with a $3 N$ vertex, shown in Fig. 1, is the following:

$$
\begin{aligned}
& \left\langle\left(j_{a} j_{b}\right)_{J}\left|2 \mathrm{~b}_{3 N}\right|\left(j_{c} j_{d}\right)_{J}\right\rangle= \\
& \sum_{h, J^{\prime}} \frac{\hat{J}^{\prime}}{\hat{J}^{2}}\left\langle\left[\left(j_{a} j_{b}\right)_{J}, j_{h}\right]_{J^{\prime}}\left|V_{3 N}\right|\left[\left(j_{c} j_{d}\right)_{J}, j_{h}\right]_{J^{\prime}}\right\rangle,
\end{aligned}
$$

The three-body matrix element (3BME) $\left\langle\left[\left(j_{a} j_{b}\right)_{J_{a b}}, j_{c}\right]_{J}\left|V_{3 N}\right|\left[\left(j_{d} j_{e}\right)_{J_{d e}}, j_{f}\right]_{J}\right\rangle$, expressed within the proton-neutron formalism, is antisymmetrized but not normalized.

We recall that the expressions in Eqs. (2) and (3) are the coefficients of the one-body and two-body terms, respectively, arising from the normal-ordering decomposition of the three-body component of a many-body Hamiltonian [37.

As mentioned in the Introduction, we include in the calculation of the $\hat{Q}$-box also the effect of second-order

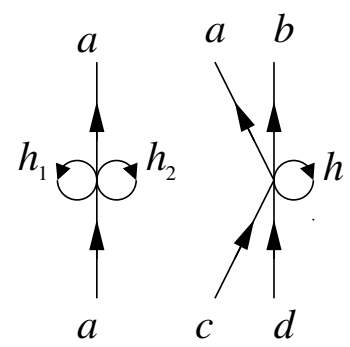

FIG. 1. First-order one- and two-body diagrams with a threebody-force vertex. See text for details.

three-body diagrams, which, for those nuclei with more than 2 valence nucleons, account for the interaction via the two-body force of the valence nucleons with core excitations as well as with virtual intermediate nucleons scattered above the model space.

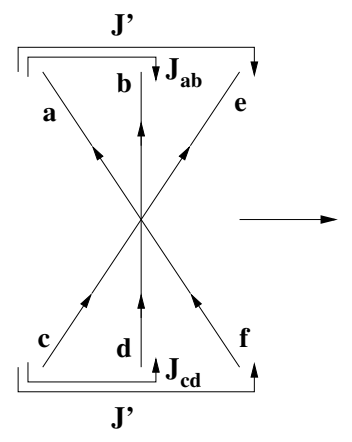

(A)

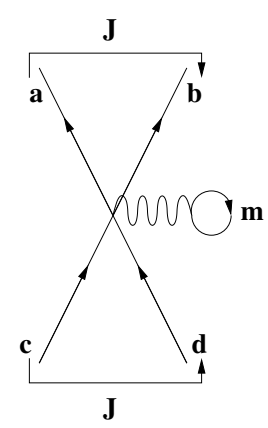

$(\alpha)$
FIG. 2. Density-dependent two-body contribution that is obtained from a three-body one. $\alpha$ is obtained by summing over one incoming and outgoing particle of the three-body graph $A$ (see text for details).

The SM code we employ 38 cannot perform the diagonalization of a three-body $H_{\text {eff }}$, so we derive from the leading-order three-body contribution a densitydependent two-body term. To this end, we calculate nine one-loop diagrams - the graph $(\alpha)$ in Fig. 2 - from the corresponding diagrams reported in Fig. 3 of Ref. 28.

Their explicit form, in terms of the three-body graph $(A)$, is the same as in Eq. 3.

$$
\begin{aligned}
& \left\langle\left(j_{a} j_{b}\right)_{J}\left|V^{\alpha}\right|\left(j_{c} j_{d}\right)_{J}\right\rangle= \\
& \sum_{m, J^{\prime}} \rho_{m} \frac{\hat{J}^{\prime}}{\hat{J}^{2}}\left\langle\left[\left(j_{a} j_{b}\right)_{J}, j_{m}\right]_{J^{\prime}}\left|V^{A}\right|\left[\left(j_{c} j_{d}\right)_{J}, j_{m}\right]_{J^{\prime}}\right\rangle
\end{aligned}
$$

where the summation over $m$-index runs in the model space and the expressions of the nine second-order diagrams $(A)$ are reported in Appendix of Ref. [28. $\rho_{m}$ 
is the unperturbed occupation density of the orbital $j_{m}$ according to the number of valence nucleons.

Finally, the perturbative expression of the $\hat{Q}$-box contains one- and two-body diagrams up to third order in the $\mathrm{N}^{3} \mathrm{LO} 2 \mathrm{NF}$ [34], one- and two-body first-order contributions in the $\mathrm{N}^{2} \mathrm{LO} 3 \mathrm{NF}$ [20, and a density-dependent two-body contribution that accounts for three-body diagrams at second-order in the $\mathrm{N}^{3} \mathrm{LO} 2 \mathrm{NF}$ [28, 29].

It should be pointed out that the latter term will lead to the derivation of specific effective shell-model Hamiltonians depending on the number of valence protons and neutrons, that obviously differ only for the two-body matrix elements.

The folded-diagram series is then summed up to all orders using the Lee-Suzuki iteration method [39].

We stress that the input chiral $2 \mathrm{NF}$ and $3 \mathrm{NF}$ have not been modified by way of any renormalization procedure, and here we will show a few details about the perturbative properties of the effective Hamiltonian. A similar discussion about the perturbative expansion of the $\hat{Q}$-box from $\mathrm{N}^{3} \mathrm{LO} 2 \mathrm{NF}$ potential has been reported in Ref. [34.

First, it should be pointed out that the truncation of the number of intermediate states appearing in the perturbative expansion is the same as in Ref. 34, i.e. the intermediate states whose unperturbed excitation energy is greater than a fixed value $E_{\max }=N_{\max } \hbar \omega$ are disregarded. As mentioned above, the value we have chosen for the HO parameter is $\hbar \omega=11 \mathrm{MeV}$. Because of our present limitation of the storage of the total number of two-body matrix elements, we can include a maximum number of intermediate states that do not exceed $N_{\max }=18$.

After these clarifying details, we present in Fig. 3 the first excited states of ${ }^{42} \mathrm{Ca}$ spectrum, which have been obtained employing $H_{\text {eff }} \mathrm{s}$ with contributions of $3 \mathrm{NF}$, and starting from $\hat{Q}$-boxes at first-, second-, and thirdorder in perturbation theory, and their Padé approximant [2|1] 40]. We employ the Padé approximant in order to obtain a better estimate of the convergence value of the perturbation series 34, as suggested in [41. The number of intermediate states is the largest we can employ, corresponding to $N_{\max }=18$.

As can be seen, the results show a very satisfactory convergence of the $H_{\text {eff }}$ with respect to the order-by-order behavior of the perturbative expansion.

We now move our focus to the issue of the dependence of $H_{\text {eff }}$ with respect to the number of intermediate states included in the calculation of second- and third-order diagrams.

In Fig. 4 they are reported the energy spectra of ${ }^{41} \mathrm{Ca}$, obtained from one-valence-neutron $H_{\text {eff }}$ s derived by employing the Padé approximant [2|1] of the $\hat{Q}$-box, and including a number of intermediates states ranging from $N_{\max }=2$ to 18 .

From the inspection of Fig. 4, it is evident that there is no sign of convergence of the single-particle spectrum of ${ }^{41} \mathrm{Ca}$ up to $N_{\max }=18$. Since the cutoff of both $2 \mathrm{NF}$ and $3 \mathrm{NF}$ is slightly larger than $2.5 \mathrm{fm}^{-1}$ and we have chosen

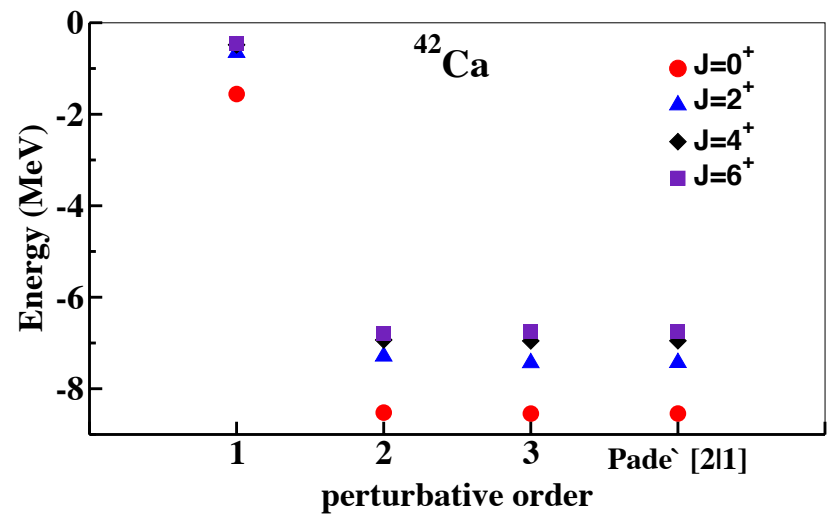

FIG. 3. Low-lying energy spectrum of ${ }^{42} \mathrm{Ca}$, obtained starting from $\hat{Q}$-boxes at first-, second-, and third-order in perturbation theory, and their Padé approximant [2|1]. See text for details.

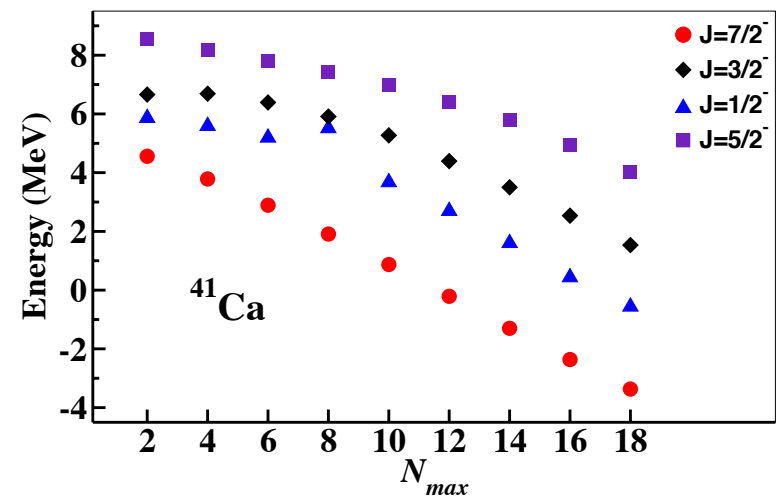

FIG. 4. Low-lying energy spectrum of ${ }^{41} \mathrm{Ca}$ relative to ${ }^{40} \mathrm{Ca}$ as a function of $N_{\max }$ (see text for details).

a value of the $\mathrm{HO}$ parameter to be equal to $11 \mathrm{MeV}$, we estimate that we need at least $N_{\max } \approx 24-26$ to reach the convergence. However, it can be clearly seen that from $N_{\max } \approx 12-14$ on the energy spacings are stable with respect to the increase in the number of intermediate states. This is an important feature, since the $H_{\text {eff }}$ for one valence-nucleon systems provides the SP energies for the SM calculations, and it is highly desirable to obtain a convergent set of theoretical SP energies to calculate excitation spectra of $f p$-shell nuclei.

Actually, the fact that the SP energies which are calculated with respect to the closed ${ }^{40} \mathrm{Ca}$ do not converge with the increasing number of intermediate states affects only the value of the ground-state energy of open-shell systems. Consequently, from now on we will employ, for our calculations, SP spacings obtained from the theory while the value of the SP energy of the $0 f_{7 / 2}$ orbital is fixed at $-1.1 \mathrm{MeV}$ for protons and $-8.4 \mathrm{MeV}$ for neutrons, consistently with experimental values of ${ }^{41} \mathrm{Sc}$ and ${ }^{41} \mathrm{Ca}$ 42 . 
After the above considerations, we move to discuss the convergence of two-valence-nucleon $H_{\text {eff }}$ with respect to the number of intermediate states. As a matter of fact, this will be a test for our theoretical TBME, since we have just observed that the SP energy spacings are convergent.

The calculated low-lying energy spectra of ${ }^{42} \mathrm{Ca}$, as a function of $N_{\max }$, are reported in Fig. 5 up to $N_{\max }=$ 18. The Padè approximant [2|1] of the $Q$-box has been calculated to derive the $H_{\text {eff }}$ s, and the theoretical SP spacings are considered relative to the experimental SP energy of the $0 f_{7 / 2}$ orbital, as mentioned before.

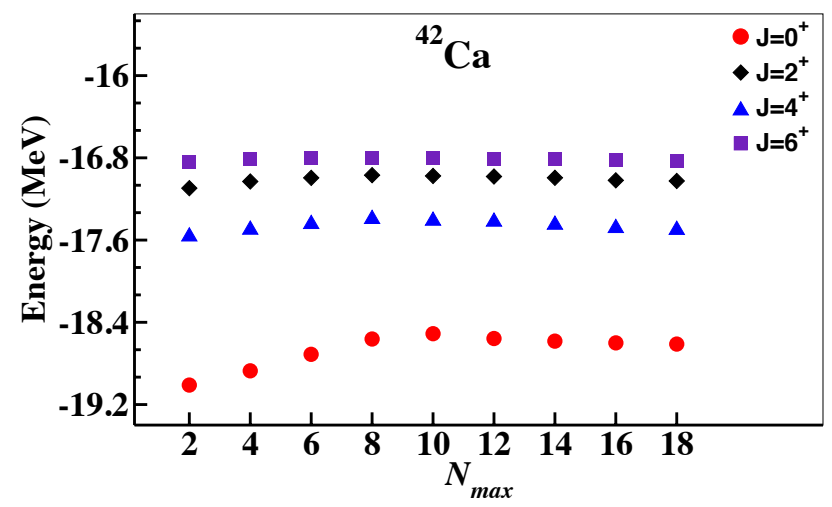

FIG. 5. Low-lying energy spectrum of ${ }^{42} \mathrm{Ca}$ as a function of the number of intermediate states included in the perturbative calculation of the $\hat{Q}$-box. See text for details.

As it happens for ${ }^{41} \mathrm{Ca}$, we observe that also the ${ }^{42} \mathrm{Ca}$ spectrum converges from $N_{\max }=12-14$ on. This leads to the conclusion that both SP spacings and TBME of our $H_{\text {eff }}$, calculated with $N_{\max }=18$, can be considered substantially stable.

Besides the convergence behavior of our $H_{\text {eff }}$, it is also important to point out that, owing to the presence of the $-U$ term in $H_{1}^{2 \mathrm{NF}}, U$-insertion diagrams arise in the $\hat{Q}$-box, and that are responsible for controlling the $\hbar \omega$ dependence introduced by the auxiliary potential $U$.

We have already addressed this issue in Ref. 34 (see Fig. 11 therein) and, in order to consider it within the present study, we show the results of the calculated yrast $J^{\pi}=2^{+}$excitation energies and two-neutron separation energies $\left(S_{2 n}\right)$ for calcium isotopes up to $N=36$ in Fig. 6. obtained with different values of the HO parameter.

The results reported in Fig. 6 have been obtained varying $\hbar \omega$ from 10 to $12 \mathrm{MeV}$. The blue bands represent the variation that is obtained if only first-order $U$-insertion diagrams are included in the calculation of the $\hat{Q}$-box, while the red bands are obtained if $U$-insertion diagrams are calculated through third order in perturbation theory.

We observe a substantial reduction of the dependence on the choice of the HO parameter as higher-order contributions of the $U$-insertion diagrams are included, in particular the closure properties at $N=28$ are very sensitive to this issue.
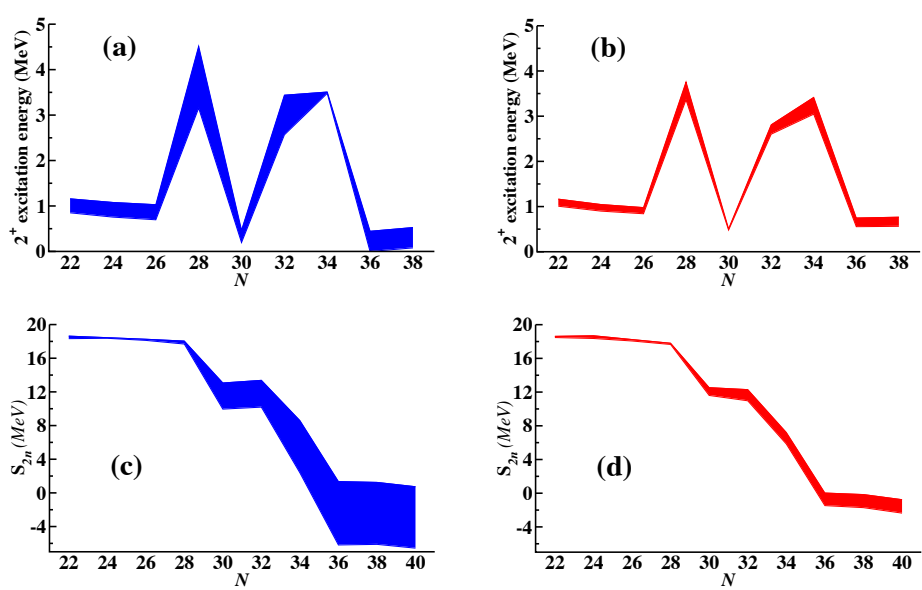

FIG. 6. Excitation energies of yrast $J^{\pi}=2^{+}$states and $S_{2 n}$ obtained for valuest of $\hbar \omega$ ranging from 10 to $12 \mathrm{MeV}$. Blue bands corresponds to the $\hat{Q}$-boxes including $U$-insertion diagrams only at first order, red bands represent results obtained including $U$-insertion diagrams up to third-order in perturbation theory. See text for details.

As mentioned before, the $H_{\text {eff }}$ derived for one-valence nucleon systems contains only one-body contributions and provides the SP energies for the SM calculation, while the two-body matrix elements are obtained from $H_{\text {eff }}$ derived from the two-valence nucleon systems, once the theoretical SP energies are subtracted from its diagonal matrix elements.

In order to perform our study, we have derived for each nucleus two classes of $H_{\text {eff }}$ s; one has been obtained calculating $\hat{Q}$-box diagrams with $2 \mathrm{NF}$ vertices only, dubbed as $H_{\mathrm{eff}}^{2 \mathrm{NF}}$. The other, indicated as $H_{\mathrm{eff}}^{3 \mathrm{NF}}$, has been built up including also $H_{1}^{3 \mathrm{NF}}$ first-order contributions in the collection of $\hat{Q}$-box diagrams (see Fig. 1). In the Supplemental Material [43] the TBMEs of $H_{\text {eff }}^{2 \mathrm{NF}}, H_{\mathrm{eff}}^{3 \mathrm{NF}}$ for systems with two valence nucleons only can be found, while the proton and neutron SP energies calculated with respect to $0 f_{7 / 2}$ orbital - $\epsilon_{\pi}$ and $\epsilon_{\nu}$ respectively - are reported in Table [

TABLE I. Theoretical proton and neutron SP energies (in $\mathrm{MeV})$ from $H_{\mathrm{eff}}^{2 \mathrm{NF}}$ and $H_{\mathrm{eff}}^{3 \mathrm{NF}}$.

\begin{tabular}{|c|c|c|c|c|}
\hline & \multicolumn{2}{|c|}{$H_{\mathrm{eff}}^{2 \mathrm{NF}}$} & \multicolumn{2}{|c|}{$H_{\mathrm{eff}}^{3 \mathrm{NF}}$} \\
\hline & $\epsilon_{\pi}$ & $\epsilon_{\nu}$ & $\epsilon_{\pi}$ & $\epsilon_{\nu}$ \\
\hline $0 f_{7 / 2}$ & 0.0 & 0.0 & 0.0 & 0.0 \\
\hline $0 f_{5 / 2}$ & 4.2 & 5.1 & 5.5 & 7.4 \\
\hline $1 p_{3 / 2}$ & 0.0 & 0.5 & 1.6 & 2.8 \\
\hline $1 p_{1 / 2}$ & 1.0 & 2.0 & 2.9 & 4.9 \\
\hline
\end{tabular}

In order to accomplish our goal to investigate the shell evolution of spectroscopic properties of $f p$ nuclei, we have performed a multipole decomposition of $H_{\mathrm{eff}}^{2 \mathrm{NF}}$ and $H_{\mathrm{eff}}^{3 \mathrm{NF}}$ for any isotope under investigation [44, 45], focussing our 
interest on their monopole components. It is worth recalling that the angular-momentum-averaged monopole component of the shell-model Hamiltonian is defined as follows:

$$
\begin{aligned}
\left\langle i, j\left|H_{\mathrm{eff}}^{m o n}\right| i, j\right\rangle & =\epsilon_{i}+\epsilon_{j}+\frac{\sum_{J}(2 J+1)\left\langle i, j\left|V_{\mathrm{eff}}\right| i, j\right\rangle_{J}}{\sum_{J}(2 J+1)}= \\
& =\epsilon_{i}+\epsilon_{j}+V_{i j}^{\text {mon }}
\end{aligned}
$$

where $V_{\text {eff }}$ is the two-body component of $H_{\text {eff }}, i$ and $j$ indicate the quantum numbers of the SP states, and the $\epsilon_{i}$ are the SP energies. Consequently, we have also studied the evolution of the proton and neutron ESPEs as a function of the valence nucleons, that are defined as:

$$
\operatorname{ESPE}(j)=\epsilon_{j}+\sum_{j^{\prime}} V_{j j^{\prime}}^{m o n} n_{j^{\prime}}
$$

where the sum runs over the model-space levels $j^{\prime}, n_{j}$ being the number of particles in the level $j$.

\section{RESULTS}

\section{A. Monopole components of the effective SM Hamiltonians}

Before we start our discussion about the characteristics of the monopole component of $H_{\mathrm{eff}}^{2 \mathrm{NF}}$ and $H_{\mathrm{eff}}^{3 \mathrm{NF}}$, it is worth coming back to the calculated SP energies of both effective Hamiltonians, which can be found in Fig. 7 as single-particle spectra of ${ }^{41} \mathrm{Sc}$ and ${ }^{41} \mathrm{Ca}$. We do not show in this figure any experimental counterpart, because the experimental information about the spectroscopic factors of both nuclei are rather scanty, and consequently we have no clear indications on the SP nature of the observed low-energy levels 46.
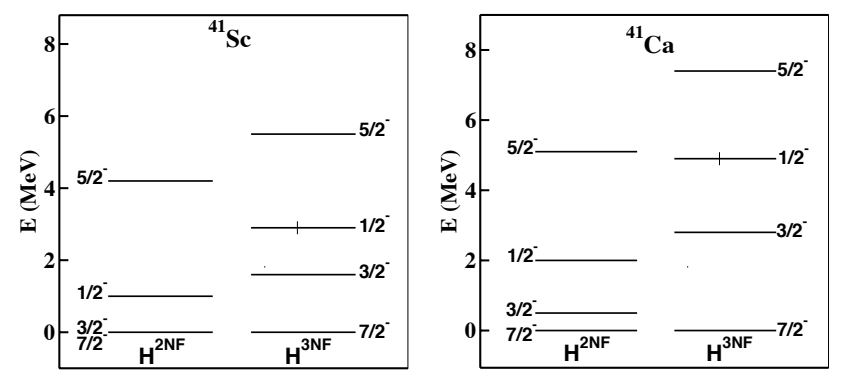

FIG. 7. Calculated SP spectra of ${ }^{41} \mathrm{Sc}$ and ${ }^{41} \mathrm{Ca}$, as obtained from $H_{\text {eff }}^{2 \mathrm{NF}}$ and $H_{\mathrm{eff}}^{3 \mathrm{NF}}$. They represent the proton and neutron $\mathrm{SP}$ energies, respectively, employed in our calculations.

From the inspection of Fig. 7. we observe that $H_{\mathrm{eff}}^{2 \mathrm{NF}}$ does not provide enough spin-orbit splitting between the $0 f_{7 / 2,5 / 2}$ orbitals in both ${ }^{41} \mathrm{Sc}$ and ${ }^{41} \mathrm{Ca}$. Moreover, the
$0 f_{7 / 2}$ and $1 p_{3 / 2}$ orbitals are not well-separated and, consequently, it can be inferred that calculations with $H_{\mathrm{eff}}^{2 \mathrm{NF}}$ might not be able to describe the shell closure that is observed at $Z, N=28$. On the other hand, the contribution coming from the $3 \mathrm{NF}$ is able to heal this defect of the SM Hamiltonian, and in the SP spectrum of $H_{\mathrm{eff}}^{3 \mathrm{NF}}$ the $0 f_{7 / 2}$ orbital is lowered enough with respect to the $1 p_{3 / 2}, 1 p_{1 / 2}, 0 f_{5 / 2}$ orbitals to lay the foundation of a better shell closure at $N, Z=28$.
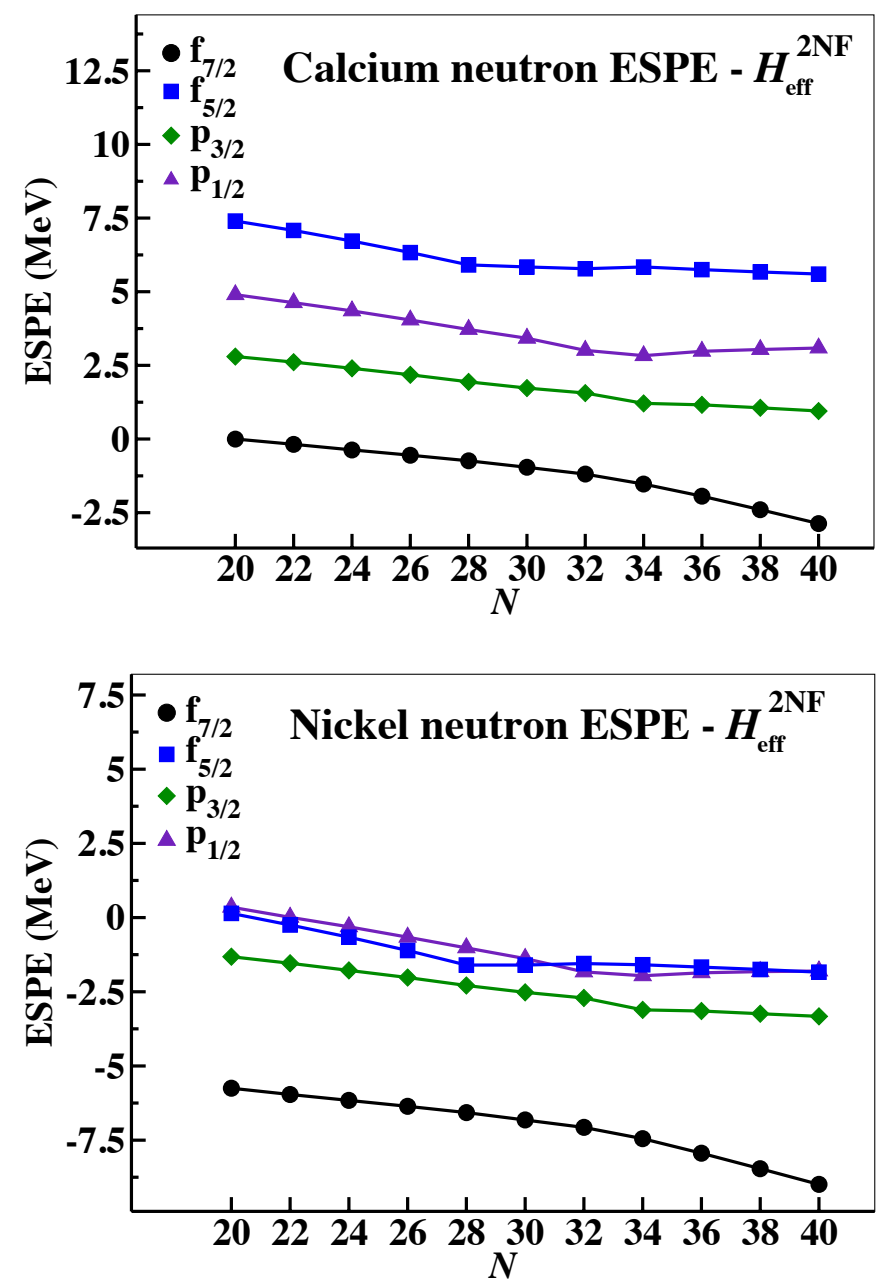

FIG. 8. Neutron ESPEs from $H_{\text {eff }}^{2 N F}$ TBMEs for calcium and nickel isotopes as a function of the neutron number (see the text for details).

Actually, a shell closure cannot be guaranteed only by the SP energy spacings, since the TBMEs of $H_{\text {eff }}$ play a crucial role in their evolution as a function of the valencenucleon number. As a matter of fact, in Ref. [20] the SP energies of $p$-shell nuclei, calculated with and without $3 \mathrm{NF}$ contributions, start both from a sufficient spinorbit splitting to provide, in principle, the $Z, N=6$ subshell closure. However, we have found that the monopole component of $H_{\mathrm{eff}}^{2 \mathrm{NF}}$ compresses the separation between the $0 p_{3 / 2}$ and $0 p_{1 / 2}$ orbitals when increasing the valence- 
nucleon number, at variance with the $H_{\mathrm{eff}}^{3 \mathrm{NF}}$ monopole term that preserves a constant energy spacing.

On the above ground, a study of the evolution of the ESPEs of $H_{\mathrm{eff}}^{2 \mathrm{NF}}$ and $H_{\mathrm{eff}}^{3 \mathrm{NF}}$ in terms of the valencenucleon number is highly desirable to understand how to obtain a sound description of their shell closure properties. This evolution of the ESPEs depends only on the TBMEs, and in the following we decide to report the neutron ESPEs of calcium isotopes and both neutron and proton ESPEs of nickel isotopes, as a function of the number of valence neutrons, calculated employing the TBMEs of $H_{\mathrm{eff}}^{2 \mathrm{NF}}$ and $H_{\mathrm{eff}}^{3 \mathrm{NF}}$, but starting from the same set of SP energies, namely those of $H_{\mathrm{eff}}^{3 \mathrm{NF}}$. This is done to evidence the relevant features of $H_{\mathrm{eff}}^{2 \mathrm{NF}}, H_{\mathrm{eff}}^{3 \mathrm{NF}}$ monopole components, and to infer their different shellevolution properties around doubly-closed ${ }^{48} \mathrm{Ca}$ and ${ }^{56} \mathrm{Ni}$.

Figure 8 shows the neutron ESPEs of calcium and nickel isotopes obtained with $H_{\mathrm{eff}}^{2 \mathrm{NF}}$ TBMEs, starting from $H_{\mathrm{eff}}^{3 \mathrm{NF}} \mathrm{SP}$ energies, and evolved as a function of the valence neutrons up to $N=40$. Black dots, blue squares, green diamonds, and indigo triangles indicate the $0 f_{7 / 2}$, $0 f_{5 / 2}, 1 p_{3 / 2}$, and $1 p_{1 / 2}$ ESPE, respectively.

As can be seen, the spacings between the $f p$ orbitals remain almost constant with respect to the evolution of the valence-neutron number, with the $0 f_{7 / 2}$ ESPE well separated from the other ones. For the calcium isotopes also $1 p_{1 / 2}, 0 f_{5 / 2}$ orbitals are separated from the $1 p_{3 / 2}$ one and between themselves too, while neutron ESPEs of nickel isotopes reveal that these three orbitals are grouped and very close to each other.

This feature seems to point to a reasonable shell closure in doubly-closed ${ }^{48} \mathrm{Ca}$ when employing the $H_{\mathrm{eff}}^{3 \mathrm{NF}}$ neutron SP spacings reported in Table $\mathrm{I}$ and TBME obtained from $H_{\mathrm{eff}}^{2 \mathrm{NF}}$, and also to a pronounced subshell closure at $N=32$ and $N=34$ for calcium isotopes. This is consistent with the results we obtained in a previous work [47, whose focus was the study of the spectroscopic properties of neutron-rich calcium isotopes. In that paper, the TBME were extracted from a $H_{\text {eff }}^{V_{\text {low }-\mathrm{k}}}$ derived from the CD-bonn potential 48 renormalized by way of the $V_{\text {low-k }}$ procedure, while the SP energies were fitted on experimental SP states in ${ }^{47,49} \mathrm{Ca}$. As a matter of fact, the role of three-body forces is mainly absorbed by the procedure of fixing SP energies to reproduce SP observables; actually, in a recent paper [49] we have shown that the theoretical SP energies obtained from $H_{\text {eff }}^{V_{\text {low-k }}}$ do not reproduce the observed shell-closure of the neutron $0 f_{7 / 2}$ orbital in ${ }^{48} \mathrm{Ca}$, the agreement between the experimental and calculated spectra of this nucleus being only qualitative.

As regards the nickel isotopes, the close values of $1 p_{3 / 2}$, $1 p_{1 / 2}, 0 f_{5 / 2}$ ESPEs may influence the shell closure in ${ }^{56} \mathrm{Ni}$ and provide the disappearance of $N=32$ and $N=34$ subshell closures.

The neutron ESPEs obtained from $H_{\text {eff }}^{3 N F}$ TBME are presented in Fig. 9 for both calcium and nickel isotopes.

The inclusion of $3 \mathrm{NF}$ effects does not affect the general behavior of the neutron ESPEs for both isotopic chains,
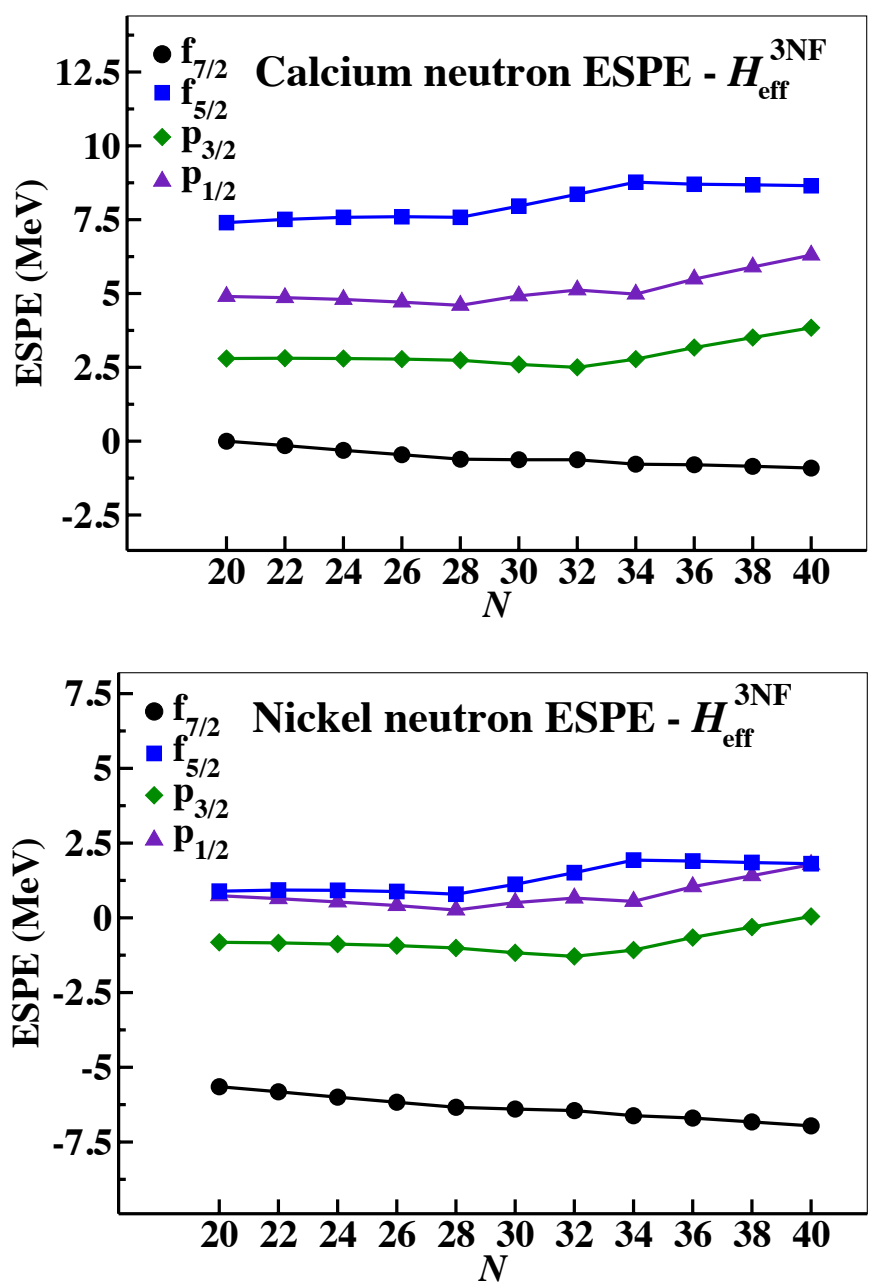

FIG. 9. Same as in Fig. 8 but for $H_{\mathrm{eff}}^{3 \mathrm{NF}}$.

but some specific details may reveal relevant features that will show up in the results of the full SM calculations in the next section.

As regards the calcium isotopes, at $N=28$ the neutron monopole component of $H_{\mathrm{eff}}^{3 \mathrm{NF}}$ enlarges the $0 f_{7 / 2}-1 p_{3 / 2}$ gap by $0.7 \mathrm{MeV}$, inducing a stronger shell closure. Also the $1 p_{1 / 2}-1 p_{3 / 2}$ and $0 f_{5 / 2}-1 p_{1 / 2}$ splittings at $N=$ 32 and $N=34$, respectively, grow and strengthen the corresponding subshell closures, as we will show in the next section.

The $3 \mathrm{NF}$ contribution to the neutron ESPEs provides also a stronger closure in ${ }^{56} \mathrm{Ni}$ since the gap between $1 p_{3 / 2}$ and $0 f_{7 / 2}$ orbitals at $N=28$ is $1 \mathrm{MeV}$ larger than the one reported in Fig. 8, that is calculated with TBME obtained from $H_{\text {eff }}^{2 \mathrm{NF}}$.

The above considerations about the ${ }^{56} \mathrm{Ni}$ shell closure are strengthened if we consider also the evolution of proton ESPEs of nickel isotopes as a function of the valenceneutron number.

As can be seen in Figs. 10, 11, the separation in energy between the $0 f_{5 / 2}$ and $0 f_{7 / 2}$ ESPEs is about $5.8 \mathrm{MeV}$ and 


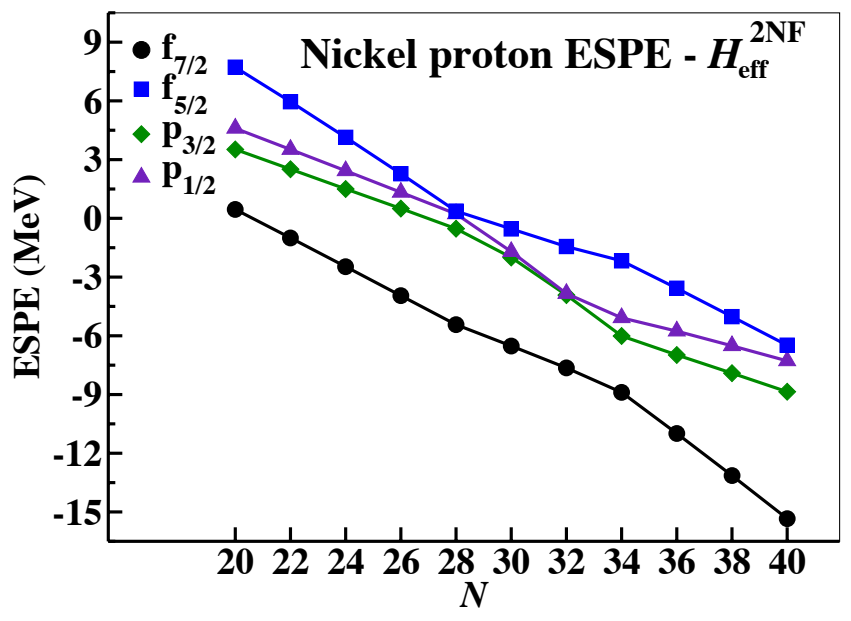

FIG. 10. Proton ESPEs from $H_{\mathrm{eff}}^{2 \mathrm{NF}}$ TBMEs for nickel isotopes as a function of the neutron number.

8.6 MeV at $N=28$, calculated with $H_{\text {eff }}^{2 \mathrm{NF}}$ and $H_{\text {eff }}^{3 \mathrm{NF}}$, respectively. Moreover, the gap between the proton ESPEs of $0 f_{5 / 2}$ and $1 p_{3 / 2}$ orbitals reduces to $0.8 \mathrm{MeV}$ at $N=28$, if only $2 \mathrm{NF}$ is considered to derive the shell-model effective Hamiltonian, while the $3 \mathrm{NF}$ contributions limit this reduction to $1.6 \mathrm{MeV}$.

These features should induce a collective effect at $N=28$, and a less pronounced shell-closure for ${ }^{56} \mathrm{Ni}$ than ${ }^{48} \mathrm{Ca}$. This collectivity affects the results obtained with $H_{\mathrm{eff}}^{2 \mathrm{NF}}$ more than those with $H_{\mathrm{eff}}^{3 \mathrm{NF}}$, as we will see in the next section.

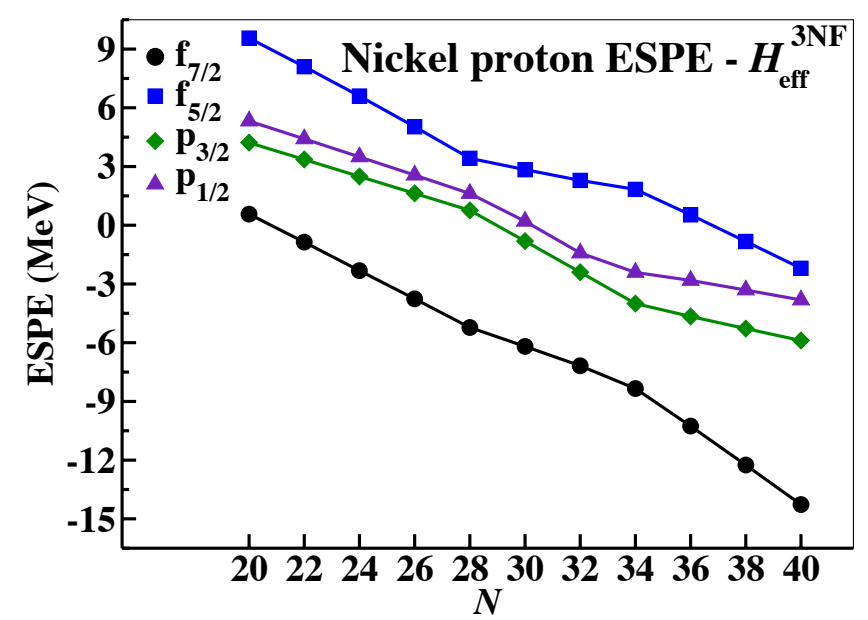

FIG. 11. Same as in Fig. 10 but for $H_{\mathrm{eff}}^{3 \mathrm{NF}}$ proton ESPEs.

\section{B. Shell-model calculations}

There are some spectroscopic features which reveal the shell closure properties, and among them two of the most important ones are the behavior of the excitation energy of $J_{1}^{\pi}=2^{+}$states and the evolution of the ground-state (g.s.) energy in even mass isotopic/isotonic chains, with respect to the number of valence neutrons/protons.

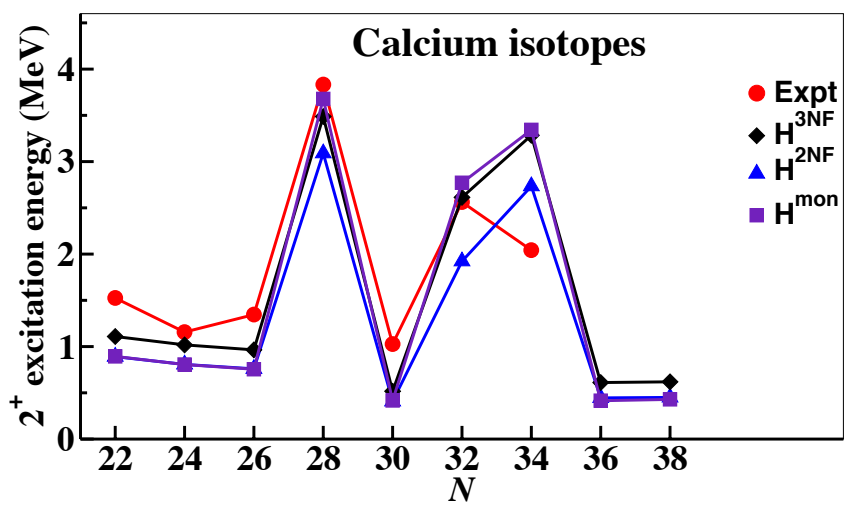

FIG. 12. Experimental and calculated excitation energies of the yrast $J^{\pi}=2^{+}$states for calcium isotopes from $N=22$ to 38 . See text for details.

These properties will be investigated by diagonalizing the two classes of Hamiltonians $H_{\mathrm{eff}}^{2 \mathrm{NF}}$ and $H_{\mathrm{eff}}^{3 \mathrm{NF}}$, and employing for both of them the set of SP energies provided by $H_{\mathrm{eff}}^{3 \mathrm{NF}}$. We refer to class of effective Hamiltonians since, as reported in Sec. III. they change according to the number of valence protons and neutrons because of the density dependence introduced by accounting for three-body correlation diagrams.

In addition to these two classes of effective SM Hamiltonians, we have built another one, that we dub $H_{\text {eff }}^{\text {mon }}$, by summing the monopole component of $H_{\mathrm{eff}}^{3 \mathrm{NF}}$ and the multipole ones belonging to $H_{\mathrm{eff}}^{2 \mathrm{NF}}$. The scope of this operation is to evidence the interplay of the monopole and multipole components through the diagonalization of the effective SM Hamiltonian, and will be better clarified in the discussion of the result of our calculations.

The experimental and theoretical results obtained with $H_{\text {eff }}^{2 \mathrm{NF}}, H_{\text {eff }}^{3 \mathrm{NF}}$, and $H_{\text {eff }}^{\text {mon }}$ will be indicated in the figures with red dots, blue triangles, black diamonds, and indigo squares, respectively.

We start our study with calcium isotopes, and in Fig. 12 they are shown the $J^{\pi}=2_{1}^{+}$excitation energies from $N=22$ up to $N=38$.

We observe that the results obtained with all three Hamiltonians are very similar. The shell closure at $N=28$ is very-well reproduced by $H_{\text {eff }}^{3 \mathrm{NF}}$ and $H_{\mathrm{eff}}^{\mathrm{mon}}$, while the $J^{\pi}=2_{1}^{+}$excitation energy obtained with $H_{\text {eff }}^{2 \mathrm{NF}}$ is about $0.7 \mathrm{MeV}$ lower than the experimental one [46]. The different results for the ${ }^{48} \mathrm{Ca}$ shell-closure trace back to the different energy gap between the $1 p_{3 / 2}$ and $0 f_{7 / 2}$ neutron ESPE when we employ the monopole term of $H_{\text {eff }}^{2 \mathrm{NF}}$ and $H_{\mathrm{eff}}^{3 \mathrm{NF}}$, as can be seen in Figs. 8 and 9

There are present also two subshell closures at $N=$ 32,34 , the second one being too strong when compared with experiment. As a matter of fact, a preliminary study of calcium isotopes, performed with a larger model space 
that includes the $0 g_{9 / 2}$ orbital too, shows that this enlargement of the model space is mandatory to reproduce the observed behavior at $N=32,34$ [50].

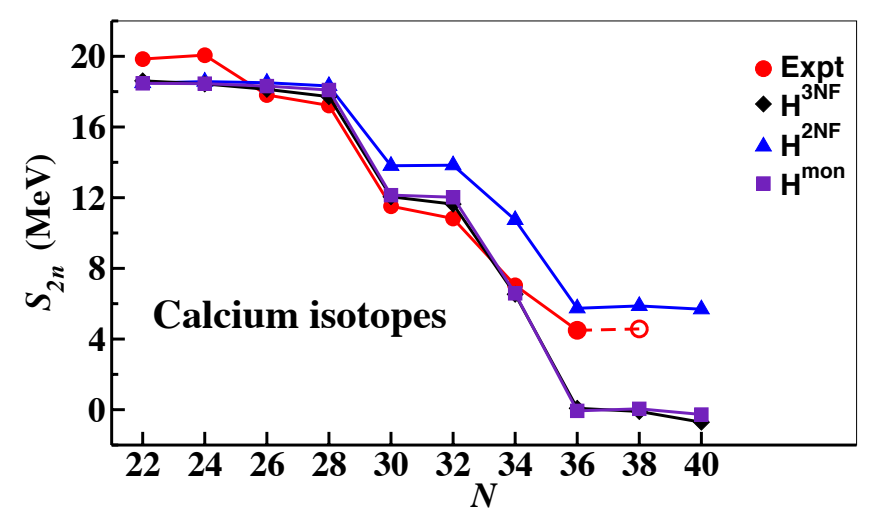

FIG. 13. Experimental and calculated two-neutron separation energies for calcium isotopes from $N=22$ to 40 . Data are taken from [42, 51, 52, open circles correspond to estimated values reported in Ref. [42. See text for details.

Different closure properties, related to whether $3 \mathrm{NF}$ are included or not in the derivation of the effective SM Hamiltonian, are present also in the calculation of the $S_{2 n}$ that are shown in Fig. 13 for the calcium isotopes up to $N=40$. As already mentioned in the previous section, we have shifted the SP energies in Table I in order to reproduce the experimental g.s. energy of ${ }^{41} \mathrm{Ca}$ and ${ }^{41} \mathrm{Sc}$ with respect to ${ }^{40} \mathrm{Ca}$.

As can be seen, both experimental $42,51,52$ and theoretical $S_{2 n}$ show a rather flat behavior up to $N=28$, then a sudden drop occurs at $N=30$ that is a signature of the shell closure due to the $0 f_{7 / 2}$ filling. Another decrease appears at $N=34$ because at that point the valence neutrons start to occupy the $1 p_{1 / 2}$ and $0 f_{5 / 2}$ orbitals.

It should be recalled that recently the comparison between experimental and calculated masses at $N=32,34$ of neutron-rich calcium isotopes has been spotted as a way to pin down the role of $3 \mathrm{NF}$ in nuclear structure calculations [15, 51.

The results obtained with $H_{\text {eff }}^{3 \mathrm{NF}}$ and $H_{\text {eff }}^{\text {mon }}$ follow closely the behavior of the experimental $S_{2 n}$ up to $N=$ 34 , while those obtained with $H_{\text {eff }}^{2 N F}$ provide a less satisfactory energy drop between $N=28$ and 30 .

At $N=36$, the repulsive $3 \mathrm{NF}$ effects contribute to a sudden drop of the two-neutron separation energies, in contrast with the experimental values. As for the case of the calculated yrast $J^{\pi}=2^{+}$excitation energies, we need to point out that a larger model space, including at least the $0 g_{9 / 2}$ orbital, improves the depiction of the spectroscopic properties of heavy-calcium isotopes [50]. Within such an enlarged model space, we have found that $H_{\text {eff }}^{3 N F}$ and $H_{\text {eff }}^{\text {mon }}$ provide a limit of the neutron dripline that is consistent with the recent observation of a bound ${ }^{60} \mathrm{Ca}$ 53, while from the inspection of Fig. 13 we observe that present results predict the calcium dripline located at $N=38$.

Now we move from systems with identical valence particle to those with both valence protons and neutrons, in order to investigate the changes in the shell evolution and closure properties originating from the collectivity ignited by the $T=0$ channel of the residual interaction.

In Fig. 14 the calculated $J^{\pi}=2_{1}^{+}$excitation energies of titanium isotopes are reported and compared with data 46. We observe that the experimental behavior is, overall, well reproduced by all three SM Hamiltonians up to $N=34$, the largest discrepancies occurring for ${ }^{42} \mathrm{Ti}$ and ${ }^{52} \mathrm{Ti}$ with all effective Hamiltonians, and for ${ }^{54} \mathrm{Ti}$ with $H_{\mathrm{eff}}^{2 \mathrm{NF}}$.

As regards the results for heavier isotopes, the underestimation of the experimental results points to the need to employ a larger model space, as already mentioned.

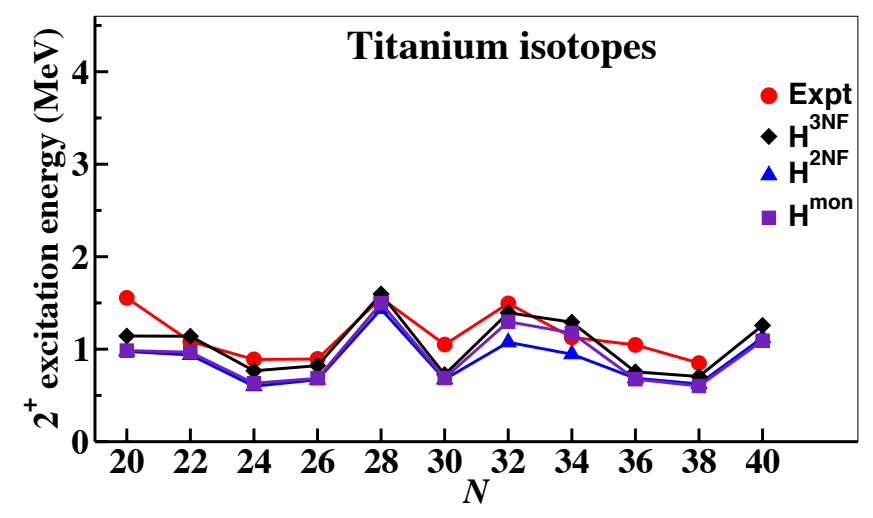

FIG. 14. Same as in Fig. 12, but for titanium isotopes from $N=20$ to 40 . See text for details.

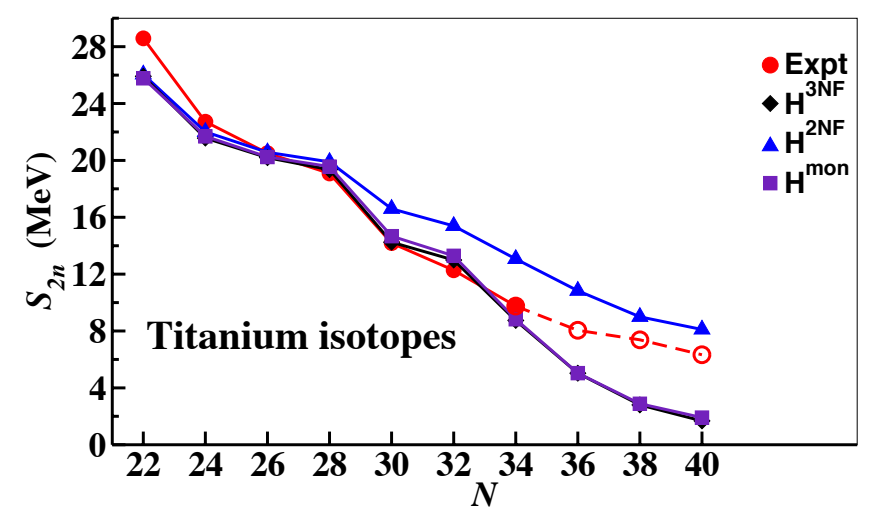

FIG. 15. Same as in Fig. 13 but for titanium isotopes from $N=22$ to 40 . See text for details.

From the inspection of Fig. 15, we observe that also the $S_{2 n}$ experimental behavior 42 is well reproduced by $H_{\mathrm{eff}}^{3 \mathrm{NF}}$ and $H_{\mathrm{eff}}^{\mathrm{mon}}$, while the calculations with $H_{\mathrm{eff}}^{2 \mathrm{NF}}$ underestimate the drop of two-neutron separation energy 
between $N=28$ and 30 . The latter feature evidences that also when the $T=0$ channel is involved, the contribution of $3 \mathrm{NF}$ helps to obtain a better comparison with experiment up to $N=34$.

The collective behavior increases with the number of interacting protons and neutrons, as can be observed for the chromium and iron isotopes. In Figs. 16 17 we report the experimental 46 and calculated excitation energies of the yrast $J^{\pi}=2^{+}$states up to $N=40$ for both isotopic chains. We observe in both cases that the calculations with $H_{\text {eff }}^{2 \mathrm{NF}}$ provide too much collectivity at $N=28$, while effective SM Hamiltonians, whose monopole component includes $3 \mathrm{NF}$ contributions, are able to reproduce the experimental behavior up to $N=34-36$ rather well.

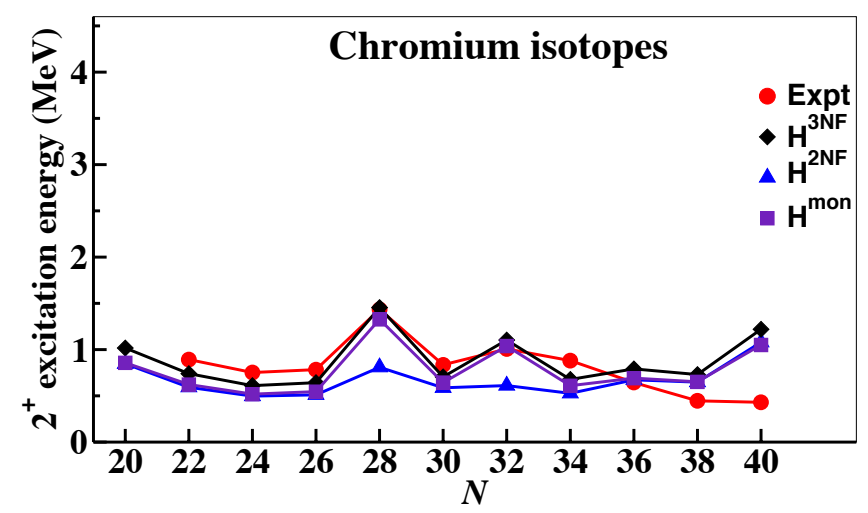

FIG. 16. Same as in Fig. 14, but for chromium isotopes. See text for details.

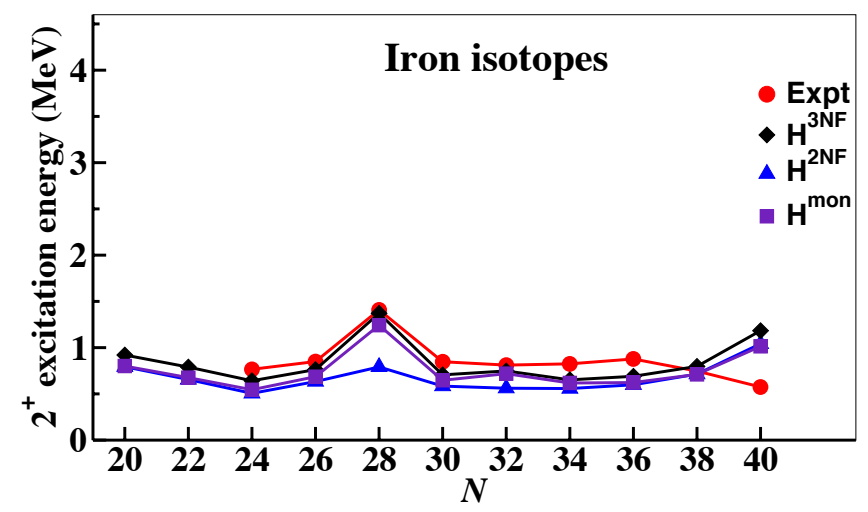

FIG. 17. Same as in Fig. 14 but for iron isotopes. See text for details.

Similar considerations follow from the inspection of Figs. 18 19, where the experimental [42] and calculated $S_{2 n}$ for chromium and iron isotopes up to $N=40$ are shown, respectively. We remind that empty red circles refer to estimated values reported in Ref. 42.

As can be seen, for these isotopes the observed $S_{2 n}$ decrease from $N=28$ to $N=30$ is no longer as steep as in calcium and titanium isotopes, evidencing the quenching of the $N=28$ shell closure.

Once again the $3 \mathrm{NF}$ contribution, which is included in the monopole component of $H_{\mathrm{eff}}^{3 \mathrm{NF}}$ and $H_{\mathrm{eff}}^{\mathrm{mon}}$, provides a better reproduction of the experimental behavior at least up to $N=34$.

Finally, we examine the nickel isotopes whose study is pivotal to understand the shell-closure properties of SM Hamiltonians. As we have seen, the proton closure at $Z=28$ is eroded by the increment of the number of valence neutrons approaching doubly-closed ${ }^{56} \mathrm{Ni}$ because of the collectivity induced by the proton-neutron interaction. Consequently, reproducing the evolution of the spectroscopic properties of nickel isotopes towards the shell closure may represent a challenging test for the theoretical SP energies and TBMEs.

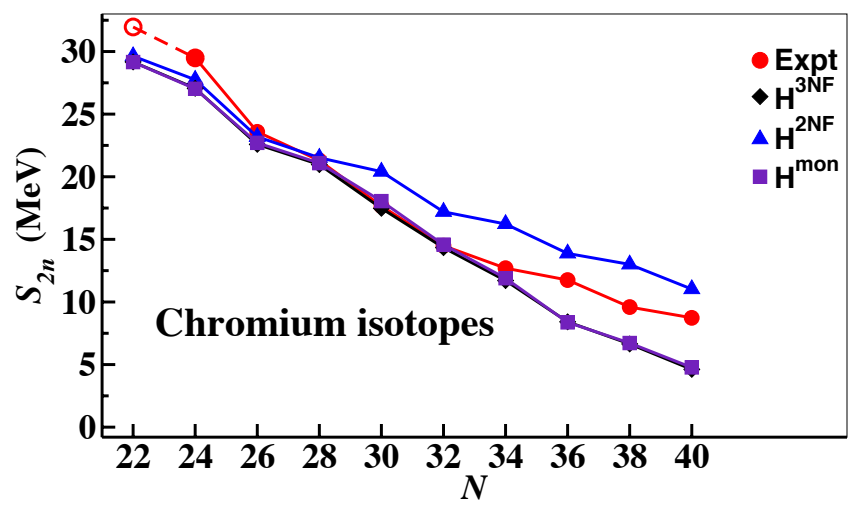

FIG. 18. Same as in Fig. 15 but for chromium isotopes. See text for details.

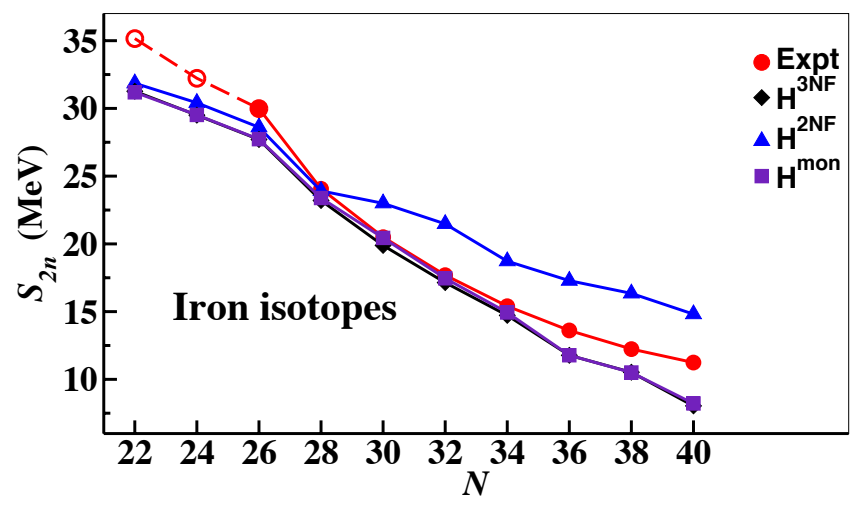

FIG. 19. Same as in Fig. 15, but for iron isotopes. See text for details.

In Fig. 20 we show the behavior of the experimental $J^{\pi}=2_{1}^{+}$excitation energies of nickel isotopes up to $N=40$ [46], and the calculated ones up to $N=38$. This different choice is due to the fact that the calculated values of the yrast $J^{\pi}=2^{+}$excitation energies for ${ }^{68} \mathrm{Ni}$ are larger than 7 and $5 \mathrm{MeV}$ with and without $3 \mathrm{NF}$ 
contribution, respectively. Such an overestimated result overshoots the energy scale of Fig. 20. we have chosen to have the same scale in all similar figures for the sake of consistency - and is a mere consequence of the limitation of $f p$-shell model space to describe heavier systems.

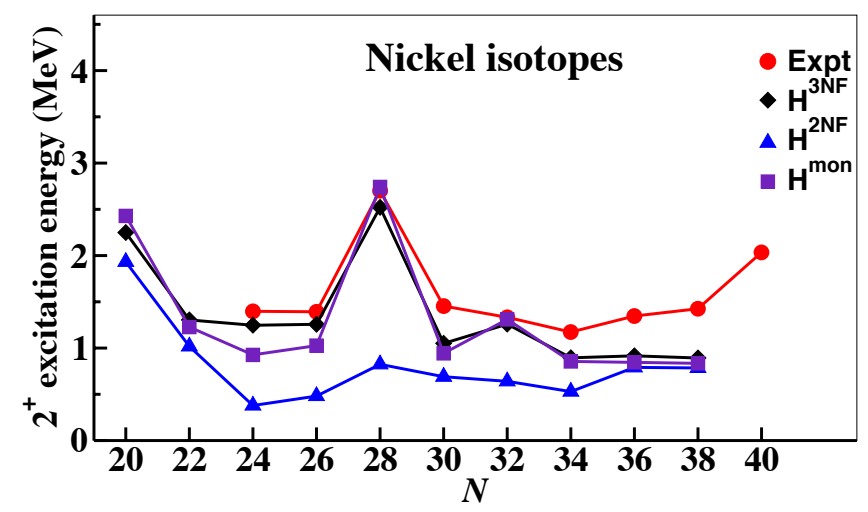

FIG. 20. Same as in Fig. 14, but for nickel isotopes. See text for details.

As can be seen, the three effective Hamiltonians predict a shell closure at $N=20\left({ }^{48} \mathrm{Ni}\right)$, although less marked with $H_{\mathrm{eff}}^{2 \mathrm{NF}}$, that confirms the ability of their monopole components to provide a similar behavior in the identical-particle channel.

Actually, both $H_{\text {eff }}^{3 N F}$ and $H_{\text {eff }}^{\text {mon }}$ results compare themselves quite well with ${ }^{52,54,56} \mathrm{Ni}$ data, while those obtained with $H_{\mathrm{eff}}^{2 \mathrm{NF}}$ exhibit a too strong collective behavior, failing to reproduce the shell closure at $N=Z=28$. As a matter of fact, the comparison between the results obtained with $H_{\mathrm{eff}}^{2 \mathrm{NF}}$ and $H_{\mathrm{eff}}^{\mathrm{mon}}$ evidences very clearly that the correct shell evolution may be obtained only including $3 \mathrm{NF}$ contributions in the monopole component of the SM Hamiltonian, the SP energies being not sufficient to balance the collectivity induced by the $T=0$ multipole component of the TBMEs.

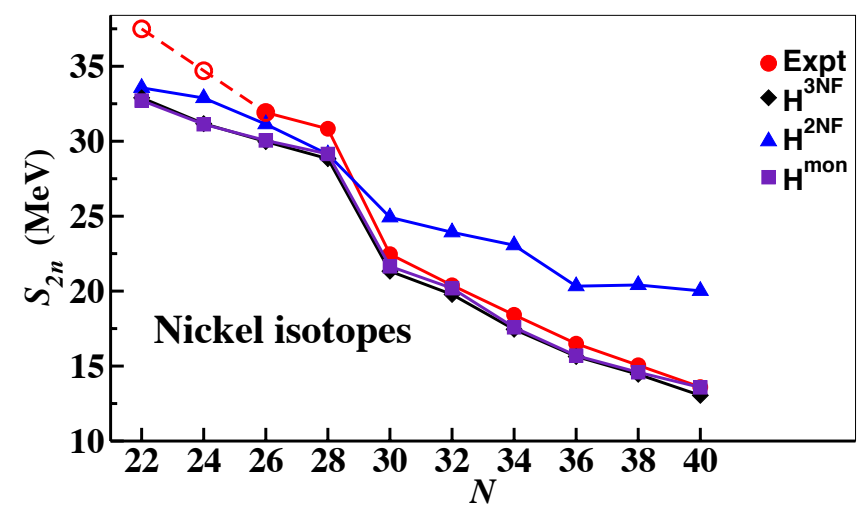

FIG. 21. Same as in Fig. 15 but for nickel isotopes. See text for details.

The same conclusions may be drawn from the inspec- tion of the behavior of the $S_{2 n}$ as a function of the valence neutrons, which are reported in Fig. 21. For nickel isotopes the drop in energy between $N=28$ and $N=30$ appears again, and the experimental behavior [42] is obtained correctly by means of $H_{\mathrm{eff}}^{3 \mathrm{NF}}$ and $H_{\mathrm{eff}}^{\mathrm{mon}}$.

We conclude our discussion about the evolution of $N=28$ shell closure summarising our results in Fig. 22 , where we have reported, for the $N=28$ isotones, the experimental and calculated behavior of both $J^{\pi}=2_{1}^{+}$ excitation energies and $B\left(E 2 ; 2_{1}^{+} \rightarrow 0_{1}^{+}\right)$transition rates. The proton and neutron effective charges to calculate the $B(E 2)$ s have been obtained by way of many-body perturbation theory using only $2 \mathrm{NF}$ vertices, and details of the derivation of effective SM one-body operators can be found in Ref. 49.

As can be seen, the filling of the proton $0 f_{7 / 2}$ orbital tunes the collectivity at $N=28$ between the doubly closed ${ }^{48} \mathrm{Ca}$ and ${ }^{56} \mathrm{Ni}$, and the evolution of such a collective behavior is well reproduced including $3 \mathrm{NF}$ contributions, but it is a failure by considering only $2 \mathrm{NF}$.

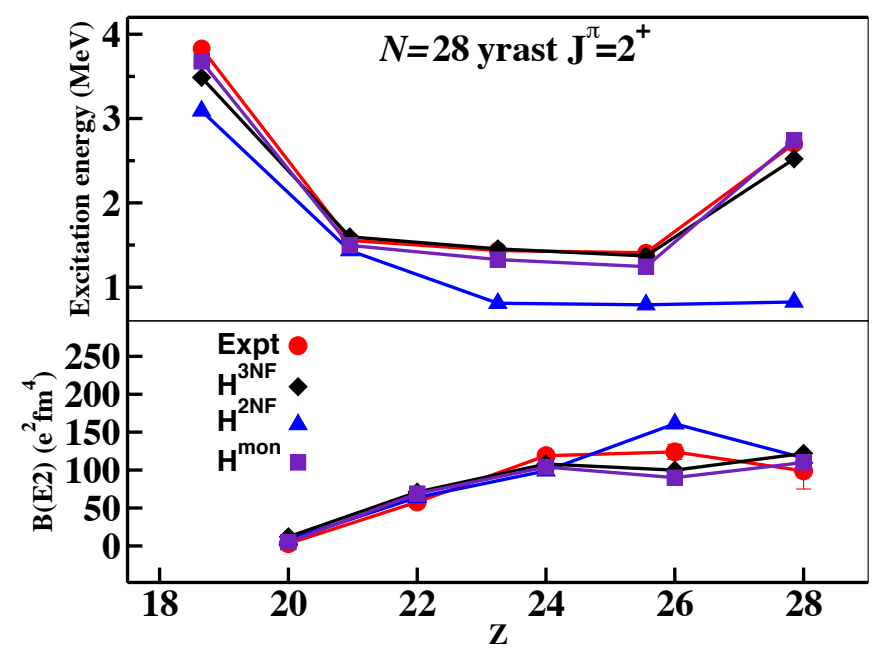

FIG. 22. Experimental and calculated excitation energies of the yrast $J^{\pi}=2^{+}$states and $B\left(E 2 ; 2_{1}^{+} \rightarrow 0_{1}^{+}\right)$transition rates for the $N=28$ isotones. See text for details.

\section{CONCLUDING REMARKS AND OUTLOOK}

In this paper we have presented the results of SM calculations for $f p$-shell nuclei in the framework of the realistic shell model, starting from chiral $2 \mathrm{NF}$ and $3 \mathrm{NF}$, and deriving effective SM Hamiltonians within the manybody perturbation theory. These effective Hamiltonians account also, in their two-body matrix elements, of the different number of valence protons and neutrons characterizing each nucleus under investigation.

In particular, we have calculated the contribution at first order in perturbation theory of a $\mathrm{N}^{2} \mathrm{LO}$ chiral $3 \mathrm{NF}$ potential to the $H_{\text {eff }}$, in order to study how it affects its monopole component and the ability to describe the 
observed shell-closure properties of $f p$ isotopic chains. To this end, starting from two different class of $H_{\text {eff }} \mathrm{S}-$ one including $3 \mathrm{NF}$ contributions and the other one not - we have first carried out an analysis of the effective single-particle energies for calcium and nickel isotopes as a function of the valence-neutron number. This study has provided information about shell-closure properties and their dependence on the $3 \mathrm{NF}$ effects included in the monopole components of $H_{\text {eff }}$.

Successively, we have performed a full diagonalization of our $H_{\text {eff }}$ for the calcium, titanium, chromium, iron, and nickel isotopes, and focussed our attention on the shell evolution of the excitation energies of the yrast $J^{\pi}=$ $2^{+}$states and the two-neutron separation energies.

The conclusion of our study can be summarised as follows:

- Starting from realistic potentials, derived within the chiral perturbation theory, the role of the $3 \mathrm{NF}$ is fundamental to obtain SP energies and TBMEs that may reproduce the shell evolution as observed from the experiment.

- The TBMEs of $H_{\text {eff }}$ derived from $2 \mathrm{NF}$ only own deficient monopole components, which cannot balance the collectivity induced by higher multipole components in the proton-neutron channel. The result is an erosion of the $N=28$ shell closure when the number of valence protons increases.
- The central role of the monopole component of the $H_{\text {eff }}$ is testified by the fact that when it is subtracted from $H_{\mathrm{eff}}^{2 \mathrm{NF}}$, and substituted with the monopole of $H_{\mathrm{eff}}^{3 \mathrm{NF}}$, the observed shell evolution and the $N=28$ shell closure is restored.

The outlook of our future work points towards the improvement of the derivation of $H_{\mathrm{eff}}^{3 \mathrm{NF}}$ by including higherorder contributions with $3 N$ vertices in the perturbative expansion of the $\hat{Q}$ box, and the investigation of heavier systems in order to assess the reliability of present approach in exotic neutron-rich nuclear systems.

\section{ACKNOWLEDGEMENTS}

This work has been supported by he National Key R\&D Program of China under Grant No. 2018YFA0404401, the National Natural Science Foundation of China under Grants No. 11835001, No. 11320101004, and No. 11575007 and the CUSTIPEN (China-US Theory Institute for Physics with Exotic Nuclei) funded by the US Department of Energy, Office of science under Grant No. de-sc0009971. We acknowledge the MARCONI Supercomputer at CINECA, under the CINECA-INFN agreement, and the High-performance Computing Platform of Peking University for providing computational resources.
[1] M. G. Mayer, Phys. Rev. 75, 1969 (1949).

[2] O. Haxel, J. H. D. Jensen, and H. E. Suess, Phys. Rev. 75, 1766 (1949).

[3] M. G. Mayer and J. H. D. Jensen, Elementary theory of nuclear shell structure (John Wiley, New York, 1955).

[4] T. T. S. Kuo and G. E. Brown, Nucl. Phys. 85, 40 (1966).

[5] E. Caurier, A. P. Zuker, A. Poves, and G. MartínezPinedo, Phys. Rev. C 50, 225 (1994).

[6] J. Duflo and A. P. Zuker, Phys. Rev. C 59, R2347 (1999).

[7] A. P. Zuker, Phys. Scr. T 157, 157 (2000).

[8] A. P. Zuker, Phys. Rev. Lett. 90, 042502 (2003).

[9] T. Otsuka, T. Suzuki, J. D. Holt, A. Schwenk, and Y. Akaishi, Phys. Rev. Lett. 105, 032501 (2010).

[10] J. D. Holt, J. Menéndez, and A. Schwenk, Eur. Phys. J. A 49, 39 (2013).

[11] K. Hebeler, J. D. Holt, J. Menéndez, and A. Schwenk, Ann. Rev. Nucl. Part. Sci. 65, 457 (2015).

[12] J. Simonis, K. Hebeler, J. D. Holt, J. Menéndez, and A. Schwenk, Phys. Rev. C 93, 011302 (2016).

[13] J. Simonis, S. R. Stroberg, K. Hebeler, J. D. Holt, and A. Schwenk, Phys. Rev. C 96, 014303 (2017).

[14] J. D. Holt, T. Otsuka, A. Schwenk, and T. Suzuki, J. Phys. G 39, 085111 (2012).

[15] J. D. Holt, J. Menéndez, J. Simonis, and A. Schwenk, Phys. Rev. C 90, 024312 (2014).

[16] S. Bogner, T. T. S. Kuo, L. Coraggio, A. Covello, and N. Itaco, Phys. Rev. C 65, 051301(R) (2002).
[17] S. Bogner, T. T. S. Kuo, and A. Schwenk, Phys. Rep. 386, 1 (2003).

[18] S. K. Bogner, R. J. Furnstahl, and R. J. Perry, Phys. Rev. C 75, 061001 (2007).

[19] S. K. Bogner, R. J. Furnsthal, and A. Schwenk, Prog. Part. Nucl. Phys. 65, 94 (2010).

[20] T. Fukui, L. De Angelis, Y. Z. Ma, L. Coraggio, A. Gargano, N. Itaco, and F. R. Xu, Phys. Rev. C 98, 044305 (2018).

[21] Y. Utsuno, T. Otsuka, T. Mizusaki, and M. Honma, Phys. Rev. C 60, 054315 (1999).

[22] E. Epelbaum, W. Glöckle, and U.-G. Meissner, Nucl. Phys. A 747, 362 (2005).

[23] R. Machleidt and D. R. Entem, Phys. Rep. 503, 1 (2011).

[24] S. Weinberg, Phys. Lett. B 295, 114 (1992).

[25] U. van Kolck, Phys. Rev. C 49, 2932 (1994).

[26] D. R. Entem and R. Machleidt, Phys. Rev. C 66, 014002 (2002).

[27] T. T. S. Kuo and E. Osnes, Lecture Notes in Physics, vol. 364 (Springer-Verlag, Berlin, 1990).

[28] A. Polls, H. Müther, A. Faessler, T. T. S. Kuo, and E. Osnes, Nucl. Phys. A 401, 124 (1983).

[29] P. J. Ellis and E. Osnes, Rev. Mod. Phys. 49, 777 (1977).

[30] P. Navrátil, V. G. Gueorguiev, J. P. Vary, W. E. Ormand, and A. Nogga, Phys. Rev. Lett. 99, 042501 (2007).

[31] P. Maris, J. P. Vary, and P. Navrátil, Phys. Rev. C 87, 014327 (2013). 
[32] J. Blomqvist and A. Molinari, Nucl. Phys. A 106, 545 (1968).

[33] M. Hjorth-Jensen, T. T. S. Kuo, and E. Osnes, Phys. Rep. 261, 125 (1995).

[34] L. Coraggio, A. Covello, A. Gargano, N. Itaco, and T. T. S. Kuo, Ann. Phys. 327, 2125 (2012).

[35] T. T. S. Kuo, S. Y. Lee, and K. F. Ratcliff, Nucl. Phys. A 176, 65 (1971).

[36] T. T. S. Kuo, J. Shurpin, K. C. Tam, E. Osnes, and P. J. Ellis, Ann. Phys. (NY) 132, 237 (1981).

[37] Lecture Notes in Physics, vol. 936 (Springer, Berlin, 2017), edited by M. Hjorth-Jensen, M. P. Lombardo, and U. van Kolck.

[38] E. Caurier, G. Martínez-Pinedo, F. Nowacki, A. Poves, and A. P. Zuker, Rev. Mod. Phys. 77, 427 (2005).

[39] K. Suzuki and S. Y. Lee, Prog. Theor. Phys. 64, 2091 (1980).

[40] G. A. Baker and J. L. Gammel, The Padé Approximant in Theoretical Physics, vol. 71 of Mathematics in Science and Engineering (Academic Press, New York, 1970).

[41] H. M. Hoffmann, Y. Starkand, and M. W. Kirson, Nucl. Phys. A 266, 138 (1976).

[42] G. Audi, A. H. Wapstra, and C. Thibault, Nucl. Phys. A 729, 337 (2003).

[43] See Supplemental material at [URL will be inserted by publisher] for the list of two-body matrix elements of the shell-model Hamiltonians $H_{\mathrm{eff}}^{2 \mathrm{NF}}, H_{\mathrm{eff}}^{3 \mathrm{NF}}$, derived for twovalence nucleon systems.
[44] A. Umeya, S. Nagai, G. Kaneko, and K. Muto, Phys. Rev. C 77, 034318 (2008).

[45] A. Umeya and K. Muto, Nucl. Phys. A 955, 194 (2016).

[46] Data extracted using the NNDC On-line Data Service from the ENSDF database, file revised as of March 15, 2019., URL https://www .nndc.bnl.gov/ensdf

[47] L. Coraggio, A. Covello, A. Gargano, and N. Itaco, Phys. Rev. C 80, 044311 (2009).

[48] R. Machleidt, Phys. Rev. C 63, 024001 (2001).

[49] L. Coraggio, L. De Angelis, T. Fukui, A. Gargano, N. Itaco, and F. Nowacki, Phys. Rev. C 100, 014316 (2019).

[50] L. Coraggio and Y. Z. Ma (2019), to be published in the Proceedings of the International Conference Nuclear Theory in the Supercomputing Era - 2018 (NTSE-2018), Daejeon, South Korea, October 29 - November 2 2018, eds. A. M. Shirokov and A. I. Mazur, Pacific National University, Khabarovsk, Russia, 2019.

[51] F. Wienholtz, D. Beck, K. Blaum, C. Borgmann, M. Breitenfeldt, R. B. Cakirli, S. George, F. Herfurth, J. Holt, M. Kowalska, et al., Nature (London) 498, 346 (2013).

[52] S. Michimasa, M. Kobayashi, Y. Kiyokawa, S. Ota, D. S. Ahn, H. Baba, G. P. A. Berg, M. Dozono, N. Fukuda, T. Furuno, et al., Phys. Rev. Lett. 121, 022506 (2018).

[53] O. B. Tarasov, D. S. Ahn, D. Bazin, N. Fukuda, A. Gade, M. Hausmann, N. Inabe, S. Ishikawa, N. Iwasa, K. Kawata, et al., Phys. Rev. Lett. 121, 022501 (2018). 